\title{
Participación ciudadana y comportamiento electoral en el Estado de México. \\ Caso: proceso electoral 2015 en el distrito 5 (Tenango del Valle) y su efecto en la calidad de la democracia
}

\author{
Citizen participation and electoral behavior in the State of Mexico. \\ Case: 2015 electoral process in district 5 (Tenango del Valle) and its \\ effect on the quality of democracy
}

JESÚS VALDÉS SÁNCHEZ

$0^{\circ}$ lus Comitiãlis / Año 4, Número 7 / enero - junio 2021 / pp. 76-90 / ISSN: 2594-1356

Recepción: 25 de mayo de 2020 / Aceptación: 21 de julio de 2020

Resumen: En México, los órganos electorales mediante la organización de elecciones determinan la distribución del poder, es decir, permiten a los actores políticos buscar objetivos que son renovados periódicamente y delimitan los recursos necesarios para llegar al máximo nivel de gobierno. En el presente escrito, se explicarán algunas de las variables por las que durante la elección de Diputados Locales y Ayuntamientos del Estado de México de 2015, en el distrito 5 Tenango del Valle, existió un nivel de competitividad alto entre las diversas fuerzas políticas, mismo que se mostrará con apoyo de la geografía y la cartografía política electoral, durante el desarrollo de la investigación.

Palabras clave: Participación ciudadana, comportamiento electoral, calidad de la democracia, Tenango del Valle, Geografía político electoral.

Abstract: In México, the electoral bodies through the organization of elections determine the distribution of power, that is, they allow political actors to seek objectives that are periodically renewed and delimit the resources necessary to reach the highest level of government. In this writing, some of the variables will be explained for which, during the 2015 election of Local Deputies and Municipalities of the State of México, in the district 5 Tenango del Valle, there was a high level of competitiveness among the various forces politics, which will be shown with support of electoral political geography and cartography, during the development of the investigation.

Key words: Citizen participation, electoral behavior, quality of democracy, Tenango del Valle, electoral political geography.

La participación electoral se ha convertido en eje de la democracia, porque el sufragio universal directo y secreto es una condición fundamental de la democracia política en el presente siglo. (Gómez, 2009) 


\section{INTRODUCCIÓN}

En México, los órganos electorales mediante la organización de elecciones determinan la distribución del poder, ${ }^{2}$ es decir, permiten a los actores políticos buscar objetivos que son renovados periódicamente y delimitan los recursos necesarios para llegar al máximo nivel de gobierno. La manera en que se manifiesta la voluntad popular se ve reflejada en el nivel de votación que obtiene cada candidata o candidato, la definición de voto nos refiere a la conversión de votos en curules. Cada vez más la sociedad demanda una reinvención de los actores políticos, la figura de representantes del pueblo ha dejado un vacío, un barril sin fondo para la ciudadanía. En este contexto, basta entender el enfoque de Zárate (2006):

Los límites reales de la democracia, [...] la decepción que padecen los electores cuando su voto no se traduce en acciones de gobierno y redundan en bienestar. Ello desemboca en un voto de castigo -para los próximos comicios ${ }^{3}$-, pero no hacia el candidato que incumplió el mandato, sino para el partido que lo postuló [...]. (p.11)

La necesidad de contar con instituciones confiables y transparentes, personas participativas e informadas y el correcto análisis de la información que permita a los interesados analizar, y determinar mediante su voto razonado a quién le darán el poder de gobernar, surge la necesidad de explicar: cómo, por qué y por quién votan las y los mexiquenses.

El propósito del presente escrito es analizar el comportamiento de las y los votantes -con apoyo de las variables de edad, sexo y nivel educativo- durante la elección de Diputados Locales y Ayuntamientos del Estado de México de 2015, en el distrito 5 Tenango del Valle; identificando las preferencias electorales en las secciones donde se generan altos índices de participación ciudadana, a través de la elaboración de mapas geográficos, con la finalidad de contribuir en la generación de análisis estadísticos que puedan ser de interés para la comunidad académica y la sociedad en general.

\section{PARTICIPACIÓN CIUDADANA Y ARQUITECTURA INSTITUCIONAL}

La necesidad de generar las condiciones mínimas de convivencia y competencia entre los actores políticos en la contienda electoral, la urgencia de contar con una institucionalidad en el gobierno capaz de representar intereses sociales y procesar el régimen

\footnotetext{
${ }^{2}$ Robert A. Pastor, sostiene que la administración electoral juega un papel importante, considerándola una variable clave para explicar el éxito o el fracaso de una transición democrática. Véase https://revistas.urosario.edu.co/xml/3596/359643444013/index.html

${ }^{3}$ Cursivas propias.
} 
PARTICIPACIÓN CIUDADANA Y COMPORTAMIENTO ELECTORAL EN EL ESTADO DE MÉXiCo. CASO: PROCESO ELECTORAL 2015 EN El Distrito 5 (TeNANGo del VALLE) Y SU EFECTO EN LA CALIDAD DE LA DEMOCRACIA

democrático de nuestro país, son condicionantes para lograr una nueva reconfiguración de nuestra democracia. Nuestra forma de gobierno requiere de normas, procedimientos, instituciones y participación activa de la ciudadanía que posibiliten la reconfiguración de nuestra democracia; además, una arquitectura institucional ${ }^{4}$ que contribuya a modelarla o desfigurarla. En palabras de Habermas (2008c):

Las luchas por el reconocimiento en el Estado democrático de derecho sólo poseen fuerza legitimadora en la medida en que todos los grupos puedan tener acceso al espacio público político, puedan oír su voz, puedan articular sus necesidades y nadie sea marginalizado o excluido (p. 160).

En este sentido, la intención de ofrecer datos cualitativos sobre el comportamiento de las y los votantes, así como la conformación política de uno de los cuarenta y cinco distritos electorales en el Estado de México, brinda información valiosa para los estudiosos de la materia de participación ciudadana, abstencionismo electoral, clientelismo electoral, partidocracia, tripartidismo, entre otras. Bajo este argumento Elizalde (2005) comenta:

[...] El principal desafío que surge de nuestro desarrollo como ciudadanos es asumir la responsabilidad por nuestro actuar, y ser capaces de entender que nuestra calidad de vida alcanza su plenitud cuando trascendemos desde nuestra conciencia individual hacia una forma de conciencia capaz de sentir como propia no solo nuestra necesidad, sino además la de cualquier otro ser humano y cualquier otra forma de vida [...] (p. 30)

La necesidad de una ciudadanía participativa e interesada en los diversos temas de la vida política del país, establece un proceso histórico y dinámico; el desarrollo del ser humano forma parte del código para desactivar la apatía ciudadana.

\section{CALIDAD DE LA DEMOCRACIA: ALGUNOS DATOS PARA CONTEXTUALIZAR}

Lograr avanzar en la calidad de la democracia a nivel subnacional, a través del análisis del comportamiento de la participación ciudadana y el ejercicio de sus derechos político-electorales, representa un reto no solo para las instituciones electorales, sino para el sistema ${ }^{5}$ político en su conjunto con el fin de recobrar la confianza de la ciudadanía.

El Informe País de 2014, ofrece datos porcentuales sobre la confianza en la institución electoral nacional encontrándose entre el 30 y 40 \%, esto nos permite iden-

\footnotetext{
${ }^{4}$ Dieter Nolhen refiere a la arquitectura institucional, como aquellos órganos electorales, normas, convenciones y formas de interacción que regulan el proceso electoral. Véase https://revistas.urosario.edu.co/xm1/3596/359643444013/index.html

${ }^{5}$ Giovanni Sartori expresa que la democracia como sistema político tiene relación con la titularidad del poder y el ejercicio del poder. Se apela al concepto de la Democracia Representativa que entiende que el poder se transmite por medio de mecanismos representativos. Para ahondar más sobre el tema véase Giovanni, Sartori (1987). Elementos de teoría política. Madrid: Alianza Editorial.
} 
tificar cómo se percibe y en qué nivel se encuentra la calidad de la democracia en el país, teniendo como indicador principal la percepción ciudadana; y de igual forma, nos da oportunidad de realizar el ejercicio hipotético de cómo se encuentra en la misma variable, la entidad mexiquense.

A efecto de mostrar algunos de los elementos que nos permitirán identificar dicho nivel, se mencionan las aportaciones de algunos intelectuales, mismos que constituyen la referencia teórica del presente trabajo de investigación.

Algunos de los elementos que constituyen una democracia procedimental, que todo régimen que se considere democrático debe considerar en su conformación según Dahl (1989), son los siguientes:

I. El derecho de voto.

II. El derecho a ser elegido.

III. El derecho de los líderes políticos a competir para conseguir apoyo y votos.

IV. Elecciones libres y justas.

V. Libertad de asociación.

VI. Libertad de expresión.

VII. Fuentes alternativas de información.

VIII. Instituciones para hacer que las políticas públicas dependan de los votos y otras expresiones de preferencia.

Una contribución de los teóricos que han analizado la calidad de la democracia, son aquellos indicadores que aportan valor agregado para este trabajo de investigación (Lijphart, 2000, pp. 262-269):

I. Representación de las mujeres (representación parlamentaria femenina, representación femenina en el gabinete). Lijphart considera que "estas son medidas importantes de la calidad de la representación democrática por derecho propio y también pueden servir como indicadores indirectos de cómo están representadas las minorías en general”.

II. Igualdad política. Medida a través de la ausencia de grandes desigualdades económicas (medida por medio de la relación ricos-pobres). Lijphart coincide con Dahl al reconocer una fuerte asociación entre la distribución como medida indirecta de desigualdad, puede considerarse al grado de alfabetismo, "ya que, a mayor porcentaje de población alfabetizada, más ampliamente serán distribuidos en la sociedad los recursos intelectuales básicos”.

III. Participación electoral. Según Lijphart "muestra hasta qué punto los ciudadanos están interesados en ser representados”.

IV. Satisfacción con la democracia. Sólo puede medirse a través de encuestas a los ciudadanos que indiquen si están satisfechos con la forma en que funciona democracia en su país, es decir, si hay más proximidad entre las políticas gubernamentales y los deseos de los votantes.

V. Proximidad gobierno-votante. Se mide la distancia entre la posición del gobierno en la escala izquierda-derecha y la posición del votante mediano.

VI. Responsabilidad y corrupción. Se mide a través del índice de corrupción. 
PARTICIPACIÓN CIUDADANA Y COMPORTAMIENTO ELECTORAL EN EL EstAdo DE MÉXico. CASO: PROCESO ELECTORAL 2015 EN El Distrito 5 (TeNANGo del VALLE) Y SU EFECTO EN LA CALIDAD DE LA DEMOCRACIA

VII. La hipótesis de John Stuart Smill. Lijphart rescata a John Stuart Mill ${ }^{6}$ quien reconoce que el gobierno de la mayoría es el requisito fundamental de la democracia. Aquí cuenta que el gabinete sea apoyado por la mayoría de los legisladores o que el ejecutivo nacional esté ocupado por un gobernante elegido por la mayoría del voto popular.

En este contexto, la gran cuestión no es el cómo importar las instituciones, sino cómo lograr que éstas evolucionen hacia sistemas institucionales renovados que incentiven la eficiencia política y económica, así como la equidad social conforme a los propios parámetros valorativos nacionales.

El estudiar los índices de participación ciudadana, para la elección de Diputados Locales y Ayuntamientos del Estado de México en el distrito 5 Tenango del Valle, durante el proceso electoral de 2015, permitirá descifrar indicadores cuantitativos de las estructuras de poder y de los cambios que la arena electoral sufre durante el ciclo electoral.

\section{COMPORTAMIENTO ELECTORAL DE LAS Y LOS VOTANTES}

Una de las variables a considerar durante el análisis del comportamiento electoral en el distrito 5 Tenango del Valle de la entidad mexiquense, es la relación que la geografía guarda entre una población y otra.

En las siguientes líneas se describe la participación ciudadana y comportamiento electoral a nivel distrital en el proceso electoral de 2015, mostrando a través de herramientas como la geografía y la cartografía política electoral, cuál fue el índice porcentual de participación ciudadana y el comportamiento de los votantes por sexo, edad y nivel educativo, en el distrito 5 Tenango del Valle. Se hace un análisis del resultado de la elección de Diputados Locales y Ayuntamientos de 2015, considerando el distrito 5 Tenango del Valle.

\section{Figura 1: Resultados electorales del distrito 5 Tenango del Valle}

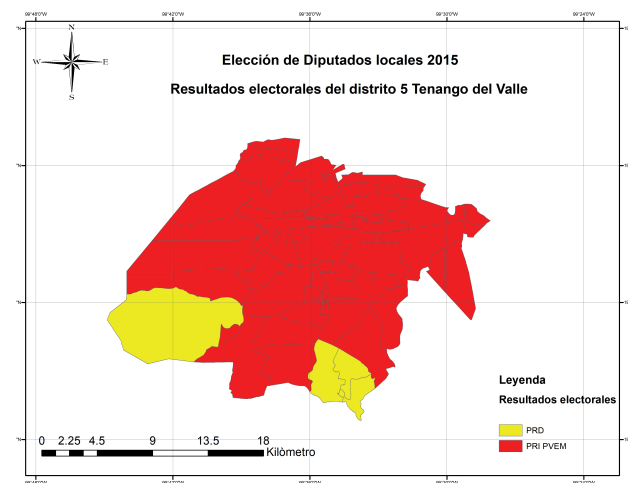

Fuente: Elaboración propia con datos del Instituto Electoral del Estado de México (2015).

\footnotetext{
${ }^{6}$ John Stuart Mill es uno de los grandes economistas clásicos, además de un sobresaliente representante de la filosofía política liberal. Véase http://www.plazayvaldes.es/autores/john-stuart-mill
} 
La Coalición ganadora en el distrito 5 Tenango de Valle, fue conformada por el Partido Revolucionario Institucional (PRI) y el Partido Verde Ecologista de México (PVEM); algo que precisar es que en las dos secciones electorales 4489 y 4495 respectivamente, el partido con mayor número de votos fue el Partido de la Revolución Democrática (PRD).

\section{Figura 2: Resultados electorales por sección del distrito 5 Tenango del Valle}

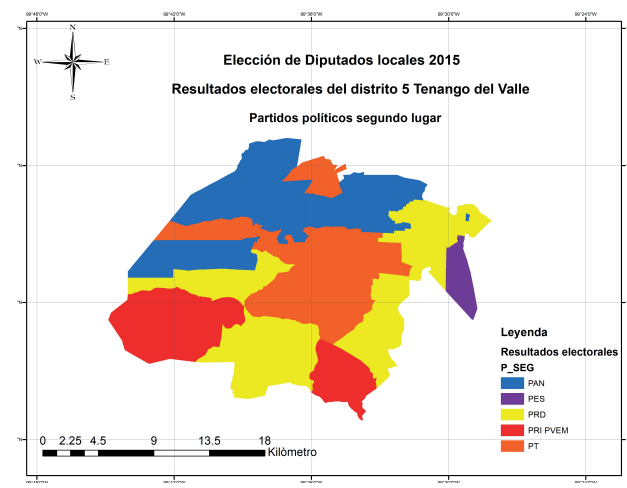

Fuente: Elaboración propia con datos del Instituto Electoral del Estado de México (2015).

Es interesante apreciar y analizar como en un mismo distrito, que corresponde a Tenango del Valle, se presenta tal nivel de pluralidad política, asimismo se muestra el escenario donde convergen ideologías de más de un partido político en los municipios que lo conforman y no sólo eso, sino que además el comportamiento de los ciudadanos al ejercer su voto por el partido político que más les genera confianza, varía entre una sección y otra. Tal es el caso del municipio mexiquense de Calimaya, donde la mayoría de las secciones las ganó (en sentido alegórico) en segundo lugar el Partido Acción Nacional (PAN) y en otras el Partido del Trabajo (PT), el primero en la sección 00501 y el segundo en la 00502, como referencia de lo anterior.

Respecto a la participación de las y los electores considerando las variables de sexo y edad, se generó el siguiente mapa; cabe mencionar, que el análisis de la información se realizó haciendo el cruce de la información del resultado obtenido por la Coalición PRI-PVEM. 
PARTICIPACIÓN CIUDADANA Y COMPORTAMIENTO ELECTORAL EN EL ESTADO DE MÉXiCo. CASO: PROCESO ELECTORAL 2015 EN El Distrito 5 (TeNANGo DEL VALLE) Y SU EFECTO EN LA CALIDAD DE LA DEMOCRACIA

Figura 3: Participación por sexo y edad de población masculina de 60 años y más

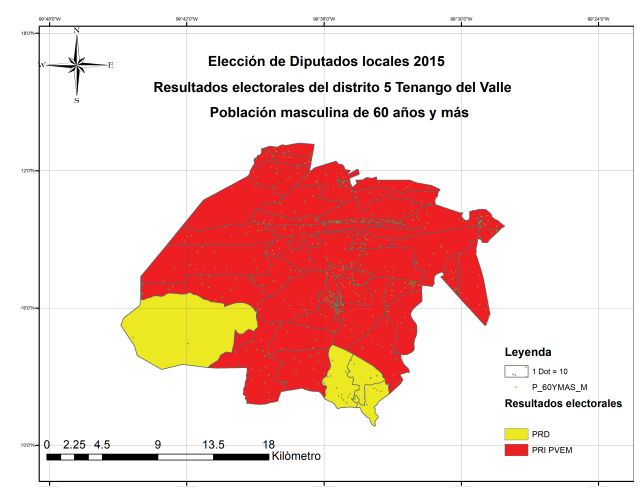

Fuente: Elaboración propia con datos del Instituto Electoral del Estado de México (2015).

Como se muestra en la figura 3, la población masculina de 60 años y más se concentra en su mayoría en los municipios del norte y este del distrito 5 Tenango del Valle, como Calimaya, San Antonio la Isla, Almoloya de Río y el municipio de Tenango del Valle, donde resultó ganadora la Coalición PRI-PVEM.

En este contexto, se puede observar de igual manera y en contraste con la concentración de la población en el sur y este del distrito, que en las secciones donde ganó el Partido de la Revolución Democrática, los votos para este partido son prácticamente nulos, su correlación es negativa.

\section{Figura 4: Correlaciones de participación por sexo y edad de población masculina} de 60 años y más

\begin{tabular}{|c|c|c|c|}
\hline & & p_60ymas_m & PRI-PVEM \\
\hline p_60ymas_m & $\begin{array}{l}\text { Correlación de Pearson } \\
\text { Sig. (bilateral) } \\
\mathrm{N}\end{array}$ & $\begin{array}{c}1 \\
6364\end{array}$ & $\begin{array}{l}.520^{* *} \\
.000 \\
6364\end{array}$ \\
\hline PRI-PVEM & $\begin{array}{l}\text { Correlación de Pearson } \\
\text { Sig. (bilateral) } \\
\mathrm{N}\end{array}$ & $\begin{array}{l}.520^{* *} \\
.000 \\
6364\end{array}$ & $\begin{array}{c}1 \\
6364\end{array}$ \\
\hline
\end{tabular}

** La correlación es significativa en el nivel 0,01 (2 colas).

Fuente: Elaboración propia con datos del Instituto Electoral del Estado de México (2015).

Ya se mencionó que la correlación con el Partido de la Revolución Democrática es negativa, por el contrario, la participación de los ciudadanos al momento de ejercer su voto en ese rango de edad, respecto a su preferencia por la Coalición es positiva, es decir, entre mayor sea el índice de participación en una elección local de ciudadanos que se encuentren en el rango de edad de 60 años y más, se verá reflejada la correlación positiva que existe con el multicitado partido político ganador; tal como 
lo muestra el siguiente gráfico:

Figura 5: Participación por sexo y edad de población masculina de 60 años y más

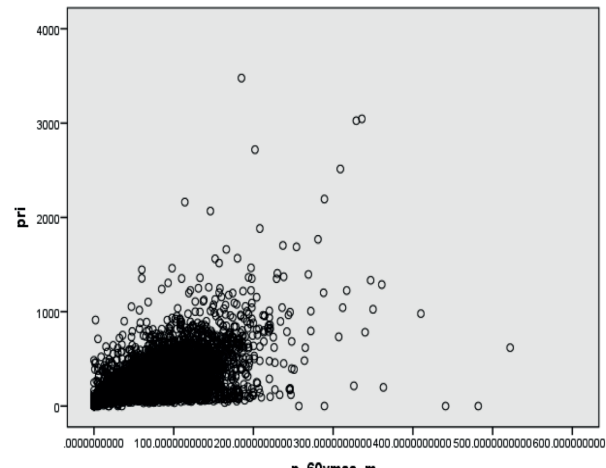

p_60ymas_m

Fuente: Elaboración propia con datos del Instituto Electoral del Estado de México (2015).

Dentro de la variable de participación por sexo y edad de población masculina, se encuentra la población masculina de $15^{7}$ a 64 años, una más de las variables de participación electoral por sexo y edad.

\section{Figura 6: Participación de población masculina de 15 a 64 años}

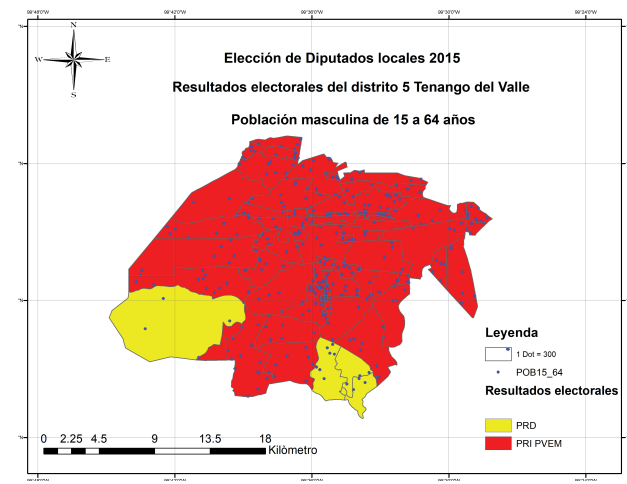

Fuente: Elaboración propia con datos del Instituto Electoral del Estado de México (2015).

Si consideramos que cada uno de los puntos representa a 300 ciudadanos distribuidos en el distrito 5 Tenango del Valle, de rebote podríamos deducir que la población masculina se concentra en el sur y este del distrito en mención, quizá este factor explique el comportamiento electoral respecto a las preferencias electorales, ya

\footnotetext{
${ }^{7}$ Recordemos que ser ciudadana o ciudadano mexicano, se deben cumplir con los requisitos que establece la Constitución Política de los Estados Unidos Mexicanos, en su Artículo 34. Véase http://www.ieem.org.mx/pdf/constitucion_1_050217.pdf, que son los siguientes: Artículo 34. Son ciudadanos de la República los varones y mujeres que, teniendo la calidad de mexicanos, reúnan, además, los siguientes requisitos: I. Haber cumplido 18 años, $y$ II. Tener un modo honesto de vivir...
} 
PARTICIPACIÓN CIUDADANA Y COMPORTAMIENTO ELECTORAL EN EL Estado DE MÉXico. CASO: PROCESO ELECTORAL 2015 EN EL Distrito 5 (TeNANGo DEL VALLE) Y SU EFECTO EN LA CALIDAD DE LA DEMOCRACIA

que los ciudadanos de ese rango de edad votaron en su mayoría por el Partido Revolucionario Institucional, en la elección de 2015. Un dato adicional, es que la participación del sector de la población en cita, donde resultó ganador el Partido de la Revolución Democrática es prácticamente nula, ahora hay que esperar el resultado del análisis de estos rangos edad del sexo femenino, sin duda arrojarán datos valiosos.

La conclusión de la participación de la población masculina de $15^{8}$ a 64 años es que existe una correlación significativa, es decir, entre más vote la población de este rango de edad, mayor será el beneficio de la Coalición PRI-PVEM, reflejado en votos.

\section{Figura 7: Participación por sexo y edad de población masculina de 15 a 64 años}

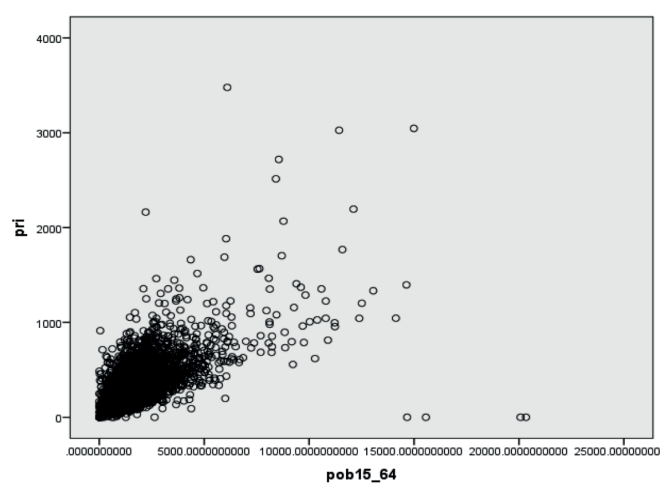

Fuente: Elaboración propia con datos del Instituto Electoral del Estado de México (2015).

La participación de la población femenina que oscila entre los $15^{9}$ y los 49 años es la siguiente:

Figura 8: Participación femenina de 15 a 49 años

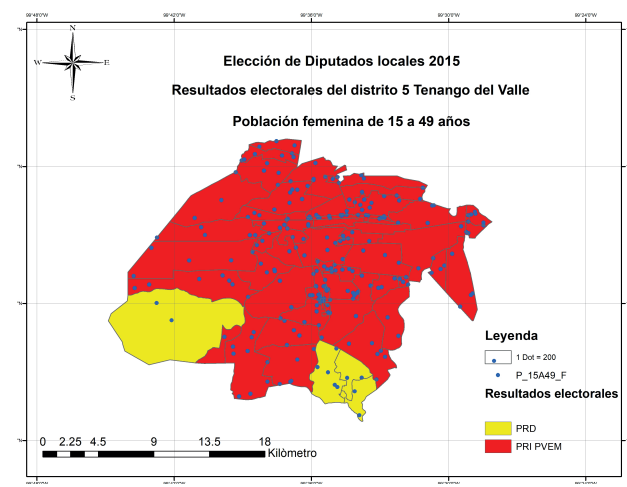

Fuente: Elaboración propia con datos del Instituto Electoral del Estado de México (2015).

\section{9 Ídem.}


Tal como lo muestra el mapa, la población femenina en este rango de edad se encuentra en el sur, este y centro del distrito 5 Tenango del Valle, analizando el resultado de la elección de Diputados Locales y Ayuntamientos, donde la Coalición PRI-PVEM se vio favorecida, podríamos resumir que la población femenina en ese rango de edad compartió preferencias por el partido político en el poder. Es decir, entre mayor sea la participación femenina, los votos por la Coalición ganadora se incrementarán, eso dice la tendencia de los datos cualitativos, tal como se muestra en la tabla de correspondencia y la gráfica.

\section{Figura 9: Correlaciones de participación por sexo y edad de población femenina de 15 a 49 años}

\begin{tabular}{|c|c|c|c|}
\hline & & PRI-PVEM & p_15a49_f \\
\hline PRI-PVEM & $\begin{array}{l}\text { Correlación de Pearson } \\
\text { Sig. (bilateral) } \\
\text { N }\end{array}$ & $\begin{array}{c}1 \\
6364\end{array}$ & $\begin{array}{l}.667^{* *} \\
.000 \\
6364\end{array}$ \\
\hline p_15a49_f & $\begin{array}{l}\text { Correlación de Pearson } \\
\text { Sig. (bilateral) } \\
\text { N }\end{array}$ & $\begin{array}{l}.667^{* *} \\
.000 \\
6364\end{array}$ & $\begin{array}{c}1 \\
6364\end{array}$ \\
\hline
\end{tabular}

** La correlación es significativa en el nivel 0,01 ( 2 colas).

Fuente: Elaboración propia con datos del Instituto Electoral del Estado de México (2015).

\section{Figura 10: Participación por sexo y edad de población femenina de 15 a 49 años}

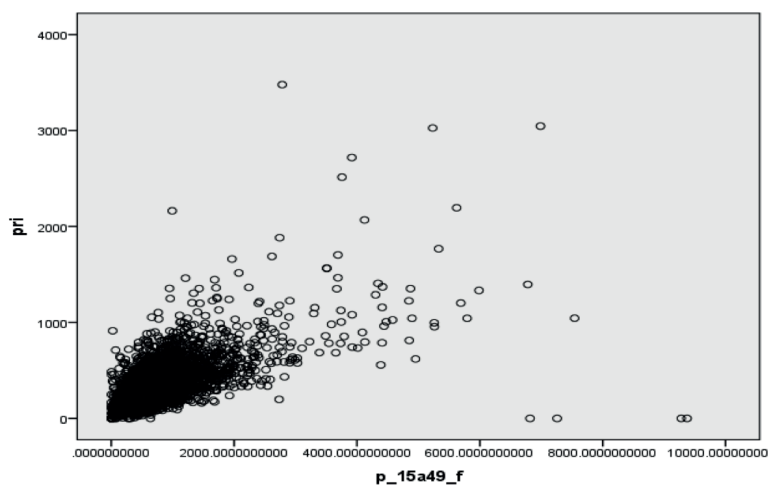

Fuente: Elaboración propia con datos del Instituto Electoral del Estado de México (2015).

Por otra parte, en el siguiente mapa se puede observar, cómo la participación femenina de 18 a 24 años se encuentra concentrada fundamentalmente en el centro y sur del distrito 5 que estamos estudiando, se muestra de qué modo la participación electoral se encuentra más dispersa en ese rango de edad, pero sigue manteniendo la hegemonía de la Coalición PRI-PVEM, de las y los electorales. 
PARTICIPACIÓN CIUDADANA Y COMPORTAMIENTO ELECTORAL EN EL EstADO DE MÉXICO. CASO: PROCESO ELECTORAL 2015 EN EL Distrito 5 (TENANGO DEL VALle) Y SU EFECTO EN LA CALIDAD DE LA DEMOCRACiA

Figura 11: Participación femenina de 18 a 24 años

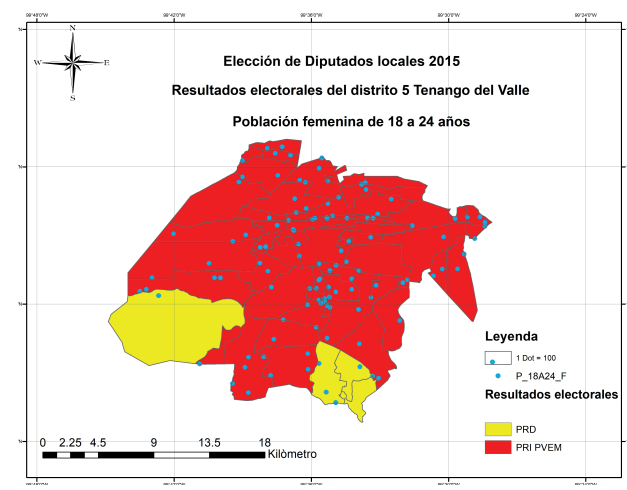

Fuente: Elaboración propia con datos del Instituto Electoral del Estado de México (2015).

Asimismo, la correlación que guarda esta variable con la participación de los electores también es significativa, podemos concluir que en el distrito 5 Tenango del Valle del Estado de México se presentó una tendencia y correlación positiva a favor de la Coalición ganadora, si retrocedemos un poco recordaremos que mencionamos que si bien es cierto que la mayoría de las secciones las gano éste partido, es evidente que los partidos políticos que ganaron el segundo lugar, con el análisis de esta información podrían focalizar de mejor manera sus programas de acción para conseguir la aceptación, preferencia y confianza de los ciudadanos, esta información es una herramienta para la calidad de la democracia.

Figura 12: Correlaciones de participación por sexo y edad de población femenina de 18 a 24 años

\begin{tabular}{cl|c|c} 
& & PRI-PVEM & p_18a24_f \\
PRI-PVEM & $\begin{array}{l}\text { Correlación de Pearson } \\
\text { Sig. (bilateral) }\end{array}$ & 1 & $\begin{array}{c}700^{* *} \\
.000 \\
6364\end{array}$ \\
\hline N & 6364 & 1 \\
\hline p_18a24_f & $\begin{array}{l}\text { Correlación de Pearson } \\
\text { Sig. (bilateral) }\end{array}$ & $\begin{array}{c}.700^{* *} \\
.000\end{array}$ & 6364 \\
\hline
\end{tabular}

${ }^{* *}$ La correlación es significativa en el nivel 0,01 ( 2 colas).

Fuente: Elaboración propia con datos del Instituto Electoral del Estado de México (2015). 
Figura 13: Participación por sexo y edad de población femenina de 18 a 24 años

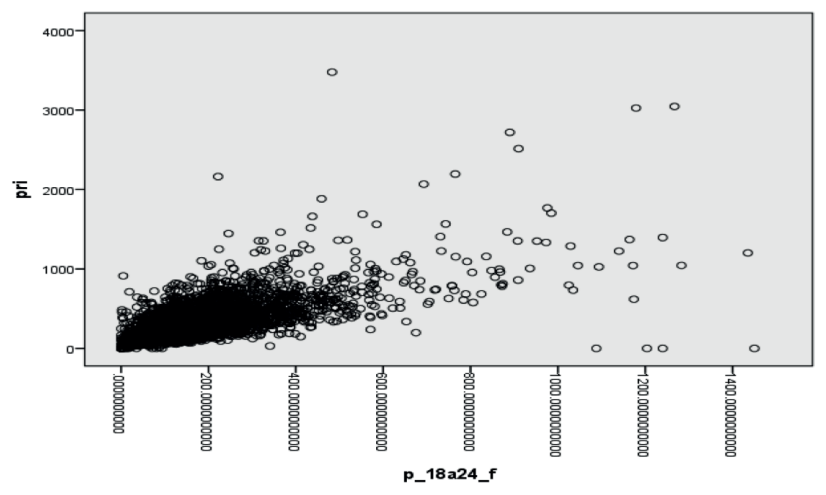

Fuente: Elaboración propia con datos del Instituto Electoral del Estado de México (2015).

La última de las variables que analizaremos en esta investigación, será de participación por sexo y grado de escolaridad.

Figura 14: Participación masculina y femenina de 18 años y más con educación pos-básica

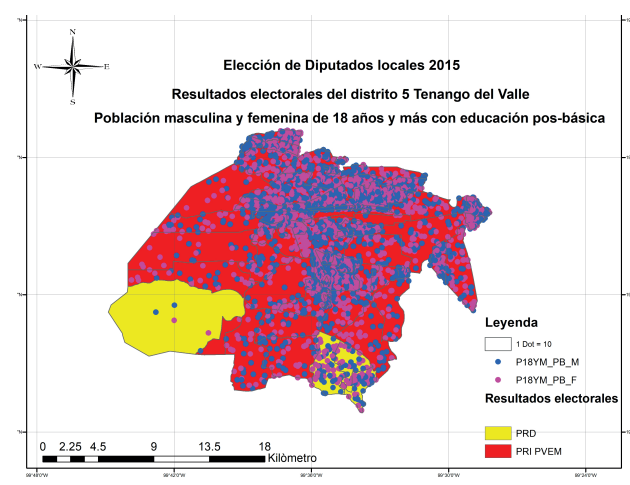

Fuente: Elaboración propia con datos del Instituto Electoral del Estado de México (2015).

Uno de los datos más significativos de la investigación es el que muestra el mapa que se está analizando, ya que el grado de escolaridad de la población masculina y femenina, nivel secundaria, básicamente se concentra entre aquellas personas que votaron por la Coalición PRI-PVEM, y justamente, se ubican en el norte y centro del distrito 5 Tenango del Valle. La tabla de correlaciones entre el partido ganador y las personas cuya edad oscila en los 18 años y más ofrece datos cualitativos de dicha aseveración. Es decir, aquellas personas que apenas cubren un nivel pos-básico tienden a votar el por el mismo partido, no importando el sexo.

En este sentido, si bien es cierto que analizando los datos en los primeros mapas, identificamos la participación y el comportamiento electoral a favor del Partido 
PARTICIPACIÓN CIUDADANA Y COMPORTAMIENTO ELECTORAL EN EL Estado DE MÉXico. CASO: PROCESO ELECTORAL 2015 EN EL Distrito 5 (TeNANGo DEL VALLE) Y SU EFECTO EN LA CALIDAD DE LA DEMOCRACIA

Revolucionario Institucional, de las personas mayores de 60 años, de igual modo, el nivel de escolaridad de nivel secundaria también se concentra en esa parte del distrito 5 Tenango del Valle.

Figura 15: Correlaciones de participación masculina y femenina de 18 años y más con educación pos-básica

\begin{tabular}{ll|c|c|c} 
& & PRI-PVEM & p18ym_pb_m & p18ym_pb_f \\
PRI-PVEM & Correlación de Pearson & 1 & $.373^{* *}$ & $.375^{* *}$ \\
& Sig. (bilateral) & 6364 & .000 & .000 \\
& $\mathrm{~N}$ & 6364 & 6364 \\
\hline \multirow{2}{*}{ p_18ym_pb_m } & Correlación de Pearson & $.373^{* *}$ & 1 & $.994^{* *}$ \\
& Sig. (bilateral) & .000 & & .000 \\
& $\mathrm{~N}$ & 6364 & 6364 & 6364 \\
\hline \multirow{2}{*}{ p_18ym_pb_f } & Correlación de Pearson & $.375^{* *}$ & $.994^{* *}$ & 1 \\
& Sig. (bilateral) & .000 & .000 & 6364 \\
\hline & $\mathrm{N}$ & 6364 & 6364 & 636 \\
\hline
\end{tabular}

** La correlación es significativa en el nivel 0,01 (2 colas).

Fuente: Elaboración propia con datos del Instituto Electoral del Estado de México (2015).

La correlación entre la población de 18 años y más, con nivel de escolaridad nivel secundaria, es positiva, lo que podemos observador en la tabla, es que guardan una correspondencia una de la otra, entre las variables independientes de edad se tiene una diferencia mínima.

\section{REFLEXIONES FINALES}

El comportamiento electoral en el Estado de México es multifactorial, la edad, el sexo y el nivel de escolaridad influyen de manera considerable al momento de emitir el voto durante las elecciones, muestra de ello es el análisis de la participación del electorado mostrado en cada una de las figuras de la investigación. La calidad de la democracia depende no solo del cumplimiento del ciclo electoral, sino del adecuado desarrollo de los procedimientos antes, durante y posterior al proceso electoral; alcanzar los resultados esperados y deseados por los distintos actores políticos, instituciones y ciudadanía, sólo puede ser logrado si se tiene claridad de la importancia de la participación ciudadana y se toman acciones transversales que integren a los diversos actores que conforman el sistema político.

Los escenarios planteados a través del estudio realizado en el distrito 5 Tenango del Valle de 2015, muestran los campos de acción para fortalecer el sistema democrático, así como la relevancia de las y los votantes en los procesos electorales. La geografía y la cartografía política electoral como herramienta para identificar y descri- 
bir la distribución de las fuerzas políticas en el Estado de México, no sólo refiere la orientación del electorado; también muestra que tendencias y posibilidades tiene un partido político de conseguir su empatía y lo posibilitan para tomar decisiones asertivas sobre sus derechos político-electorales.

Por último, resulta interesante conocer el comportamiento que las y los ciudadanos que emitieron su voto en la elección de Diputados Locales y Ayuntamientos del Estado de México de 2015, a nivel seccional y en un distrito con la densidad poblacional y la participación ciudadana de Tenango del Valle; es un ejercicio que se debería replicar en cada uno de los municipios del Estado de México, tema que debe ser prioridad para los partidos políticos, pues este tipo de trabajo ofrece datos estadísticos valiosos sobre el comportamiento electoral y la tendencia en las elecciones; por otra parte, nos permite conocer la geografía del territorio estatal, su conformación política y los posibles escenarios que pueden presentarse en la construcción de la democracia en la entidad mexiquense.

\section{REFERENCIAS}

1. Arreola Ayala, Álvaro (Coord.) (2003). Comportamiento político y tendencias electorales en el estado de México. México: Instituto Electoral del Estado de México.

2. Cansino, Cesar y Covarrubias, Israel (2007). Por una democracia de calidad. México después de la transición. México: Centro de Estudios de Político Comparada.

3. Dahl, Robert. (1989). La Poliarquía. Madrid: Tecnos.

4. Elizalde Hevia, Antonio (2005). Desarrollo humano y ética para la sustentabilidad. Santiago de Chile: Universidad Bolivariana / Universidad de Antioquia.

5. Faustino Torres, Antonio (2016). Los órganos electorales administrativos en México y los problemas de su autonomía: los casos del IEDF y del IEEM. Toluca: Instituto Electoral del Estado de México.

6. Gómez Tagle, Silvia (2009). ¿Cuántos votos necesita la democracia? La participación electoral en México 1961-2006. México: Instituto Federal Electoral.

7. Habermas, Jürgen (2008c). “Una conversación sobre cuestiones de teoría política”. En Más allá del Estado nacional (pp. 145-166). Madrid: Trotta.

8. Lijphart, Arent., (2000). Modelos de Democracia. Formas de gobierno y resultados en treinta y seis países. Barcelona: Ariel.

9. López Montiel, Gustavo; Mirón Lince, Rosa María y Reveles Vázquez, Francisco (Coords.) (2009). Los estados en el 2009: lucha política y competencia electoral. México: Instituto Electoral del Estado de México. 
PARTICIPACión CIUDADANA Y COMPORTAMIENTO ELECTORAL EN EL Estado DE MÉXICO. CASO: PROCESO ELECTORAL 2015 EN EL Distrito 5 (TeNANGo DEL VALLE) Y SU EFECTO EN LA CALIDAD DE LA DEMOCRACIA

10. López Montiel, Gustavo; Mirón Lince, Rosa María y Reveles Vázquez, Francisco (Coords.) (2012). Los estados en 2012: alternancias y hegemonías. México: Tecnológico de Monterrey.

11. Méndez de Hoyos, Irma (2016). "Integridad electoral y órganos electorales en México: elecciones 2015”. Primer Foro nacional de administración electoral y calidad de las elecciones en México. México: Flacso.

12. Nohlen, Dieter (2016). Arquitectura institucional, contexto sociocultural e integridad electoral. Desafios, Issue 28, 429-453.

13. Sartori, Giovanni (1987). Elementos de teoría política. Madrid: Alianza Editorial.

14. Sartori, Giovanni (1987). Elementos de teoría política. Madrid: Alianza.

15. Silvia Gómez Tagle (1997). La transición inconclusa. Treinta años de elecciones en México. México: El Colegio de México.

16. Villarreal Martínez, Juan C. (2012). Monografía participación y resultados electorales en el estado de México 1996-2012. México: Instituto Electoral del Estado de México.

17. Zarate Flores, Alfonso (2006). Abstencionismo, crisis de partido y fragilidad democrática. México: Instituto Electoral del Estado de México. 